\title{
COMPORTAMENTO FISIOLÓGICO DE SEMENTES DE TANGERINA (Citrus reticulata Blanco) SUBMETIDAS À DESIDRATAÇÃ̃ ${ }^{1}$
}

\author{
LEILA MARTINS ${ }^{2} \&$ WALTER RODRIGUES DA SILVA ${ }^{3}$
}

\begin{abstract}
RESUMO - O objetivo desta pesquisa foi o de estudar os efeitos imediatos da desidratação sobre o comportamento fisiológico das sementes de tangerina (Citrus reticulata Blanco var. Cleópatra). Assim, sementes extraídas manualmente de frutos maduros foram lavadas em água corrente e drenadas à sombra. Em seguida, uma porção representativa de sementes foi removida a fim de constituir o tratamento com o maior grau de umidade $(49 \%)$ a ser estudado. As sementes remanescentes foram submetidas à desidratação em equipamento com circulação forçada de ar $\left(28 \pm 2^{\circ} \mathrm{C}\right)$, visando à obtenção dos tratamentos $\left(39 ; 32 ; 24 ; 13 ; 10\right.$ e 7\% $\left.\mathrm{H}_{2} \mathrm{O}\right)$ referentes aos demais graus de umidade desejados. Foram realizadas avaliações do grau de umidade, da germinação, da taxa e da velocidade de emergência das plântulas, dos comprimentos da raiz, do hipocótilo e da plântula. Concluiuse que o potencial fisiológico das sementes, considerando o intervalo de 49 a $7 \%$ para o grau de umidade, é favorecido pela desidratação até $39 \%$ $\mathrm{H}_{2} \mathrm{O}$.
\end{abstract}

Termos para Indexação: dessecação, semente recalcitrante.

\section{PHYSIOLOGICAL PERFORMANCE OF TANGERINE (Citrus reticulata Blanco) SEEDS SUBMITTED TO DEHYDRATION}

\begin{abstract}
The objective of this research was to study the immediate effects of dehydration on the physiological performance of 'Cleopatra' tangerine (Citrus reticulata Blanco) seeds. Seeds were hand extracted from mature fruits, washed in running water and then drained in the shade. Following that, a representative portion of the seeds was removed so as to constitute the treatment containing the highest water content (49\%) to be studied. The remaining seeds were submitted to dehydration in an equipment with forced air flow $\left(28^{\circ} \pm 2^{\circ} \mathrm{C}\right)$ in order to obtain the treatments $(39$; $32 ; 24 ; 13 ; 10$ and $7 \% \mathrm{H}_{2} \mathrm{O}$ ) relative to the other moisture contents desired. Evaluations were made of the moisture level, the germination, the seedling emergence, and root, hypocotyl and seedling length. It was concluded that the physiological potential of the seeds, considering the interval of 49 to $7 \%$ moisture content, is favored by dehydration to $39 \% \mathrm{H}_{2} \mathrm{O}$.
\end{abstract}

Index Terms: dehydration, recalcitrant seed.

\section{INTRODUÇÃO}

Na maioria das espécies, as sementes podem ser desidratadas sem perdas apreciáveis de vigor (Harrington, 1972; Toledo \& Marcos Filho, 1977; Carvalho et al., 2002), havendo indicações de que a qualidade fisiológica, durante o armazenamento, seja mais afetada pelo grau de umidade das sementes do que pelos métodos de secagem a que foram submetidas (Araújo et al., 1989; Barboza \& Herrera, 1990; Vasconcelos et al., 1992). No entanto, em sementes recalcitrantes, a desidratação favorece a deterioração (Farrant et al., 1988) similarmente ao relatado por Nakamura (1975), para as sementes de citros.

King \& Roberts (1979) classificaram as sementes de citros, quanto à tolerância à secagem, como intermediárias e recalcitrantes. Hong \& Ellis (1995) relataram que, de maneira generalizada, a manutenção do teor de água em torno de $40 \%$, em sementes de citros, possibilitou a manutenção da viabilidade por vários anos. Segundo Roberts (1972), sementes de Citrus aurantium L. (laranja-azeda) submetidas à dessecação de 44 para 3,8\% $\mathrm{H}_{2} \mathrm{O}$ tiveram a qualidade fisiológica reduzida de 90 para 22\%. Entretanto, Mumford \& Panggabean (1982) observaram perda total de viabilidade, após cinco dias, pela dessecação abaixo de $8 \% \mathrm{H}_{2} \mathrm{O}$.

Os trabalhos disponíveis a respeito das conseqüências da dessecação nas sementes de Citrus reticulata Blanco são escassos e, portanto, há a necessidade de intensificação na busca de informações sobre o tema. Dessa forma, o objetivo desta pesquisa foi o de estudar os efeitos imediatos da desidratação sobre o comportamento fisiológico das sementes de tangerina.

\section{MATERIAL E MÉTODOS}

A pesquisa, realizada no Laboratório Central de Sementes e Mudas do Departamento de Sementes, Mudas e Matrizes da Coordenadoria de Assistência Técnica Integral (Campinas-SP), empregou sementes de tangerina (Citrus reticulata Blanco) da variedade Cleópatra que, extraídas manualmente de frutos maduros, foram lavadas em água corrente e drenadas à sombra. Em seguida, foi determinado o grau de umidade (Brasil, 1992) das sementes e removido o tratamento com o maior grau de umidade $(49 \%)$ a ser estudado. As sementes remanescentes foram submetidas à secagem, em equipamento com circulação forçada de $\operatorname{ar}\left(28 \pm 2^{\circ} \mathrm{C}\right)$, visando à obtenção dos tratamentos $\left(39 ; 32 ; 24 ; 13 ; 10\right.$ e $\left.7 \% \mathrm{H}_{2} \mathrm{O}\right)$ referentes aos demais graus de umidade desejados.

Esses tratamentos foram obtidos através do acompanhamento da perda de massa das sementes durante a desidratação; para tanto, amostras de sementes destinadas ao monitoramento, com massas iniciais previamente conhecidas, foram acondicionadas em sacos de filó e distribuídas nas bandejas do secador para pesagens a intervalos regulares. As massas finais das amostras, correspondentes a cada um dos graus de umidade desejados, foram calculadas utilizando a equação descrita por Cromarty et al. (1985):

$\mathrm{Mf}=\mathrm{Mi}(100-\mathrm{Ui}) \mathrm{x}(100-\mathrm{Uf})-1$

onde:

Mf = massa da amostra $(\mathrm{g})$ após a secagem;

$\mathrm{Mi}=$ massa da amostra $(\mathrm{g})$ antes da secagem;

$\mathrm{Ui}=$ grau de umidade $(\%)$ antes da secagem;

$\mathrm{Uf}=$ grau de umidade $(\%)$ desejado após a secagem.

À medida que foram sendo atingidos graus de umidade próximos aos desejados, amostras foram retiradas, homogeneizadas e divididas em frações que, por sua vez, foram embaladas em sacos de polietileno $(0,14 \mathrm{~mm}$ de espessura $)$ e mantidas a $10 \pm 2^{\circ} \mathrm{C}$, provisoriamente, até a obtenção de todos os tratamentos.

Foram realizadas as seguintes avaliações:

a) Determinação do grau de umidade: foi conduzido a $105 \pm 3^{\circ} \mathrm{C} /$ 24h, pelo método da estufa (Brasil, 1992), em duas amostras de 25 sementes por repetição. Os resultados obtidos, com base na massa úmida, foram expressos em porcentagem.

\footnotetext{
${ }^{1}$ (Trabalho 097/2005). Recebido: 14/06/2005. Aceito para publicação: 08/03/2006. Apoiado financeiramente pela Fundação de Amparo à Pesquisa do Estado de São Paulo (FAPESP).

${ }^{2}$ Laboratório Central de Sementes e Mudas, DSMM - CATI. Caixa Postal 1291, CEP 13073-001 Campinas (SP), Brasil / E-mail: leila@cati.sp.gov.br ${ }^{3}$ In memoriam.
} 
TABELA 1 - Classificação estatística: exemplo hipotético das pontuações parciais, obtidas em cada avaliação fisiológica, e total ( $\sum$ das pontuações parciais) atribuídas aos tratamentos.

\begin{tabular}{|c|c|c|c|c|c|}
\hline \multirow[b]{2}{*}{ Tratamentos } & \multicolumn{2}{|c|}{ Avaliação x } & \multicolumn{2}{|c|}{ Avaliação y } & \multirow[b]{2}{*}{ Pontuação total } \\
\hline & Dados & Pontuação parcial & Dados & Pontuação parcial & \\
\hline 2 & $99 \mathrm{ab}$ & $(+1)+(0)=+1$ & $97 \mathrm{bc}$ & $(0)+(-1)=-1$ & 0 \\
\hline 4 & 89 bc & $(0)+(-1)=-1$ & $96 \mathrm{c}$ & $(0)+(-3)=-3$ & -4 \\
\hline 5 & $87 \mathrm{c}$ & $(0)+(-2)=-2$ & $99 \mathrm{ab}$ & $(+1)+(0)=(+1)$ & -1 \\
\hline
\end{tabular}

TABELA 2 - Dados do teor de água (U), da germinação (G), da taxa (E) e da velocidade (IVE) de emergência das plântulas, dos comprimentos da raiz $(\mathrm{CR})$, do hipocótilo $(\mathrm{CH})$ e da plântula $(\mathrm{CP})$ obtidos em sementes de tangerina submetidas à desidratação $(1)$.

\begin{tabular}{ccllllll}
\hline Tratamentos (Grau de umidade, \%) & $\mathrm{U}(\%)$ & $\mathrm{G}(\%)$ & $\mathrm{E}(\%)$ & $\mathrm{IVE}$ & $\mathrm{CR}(\mathrm{cm})$ & $\mathrm{CH}(\mathrm{cm})$ & $\mathrm{CP}(\mathrm{cm})$ \\
\hline 49 & 49,2 & $72,5 \mathrm{ab}$ & $27,5 \mathrm{a}$ & $0,71 \mathrm{ab}$ & $1,63 \mathrm{ab}$ & $1,21 \mathrm{a}$ & $2,84 \mathrm{ab}$ \\
39 & 39,2 & $85,5 \mathrm{a}$ & $28,0 \mathrm{a}$ & $1,25 \mathrm{a}$ & $1,98 \mathrm{a}$ & $1,08 \mathrm{a}$ & $3,06 \mathrm{a}$ \\
32 & 31,8 & $71,0 \mathrm{ab}$ & $18,0 \mathrm{ab}$ & $0,72 \mathrm{ab}$ & $1,44 \mathrm{ab}$ & $0,97 \mathrm{ab}$ & $2,41 \mathrm{ab}$ \\
24 & 24,1 & $59,0 \mathrm{bc}$ & $11,0 \mathrm{ab}$ & $0,30 \mathrm{bc}$ & $0,56 \mathrm{bc}$ & $0,41 \mathrm{ab}$ & $0,97 \mathrm{abc}$ \\
13 & 13,3 & $45,5 \mathrm{c}$ & $14,5 \mathrm{ab}$ & $0,39 \mathrm{bc}$ & $0,85 \mathrm{abc}$ & $0,56 \mathrm{ab}$ & $1,41 \mathrm{abc}$ \\
10 & 10,2 & $53,0 \mathrm{bc}$ & $8,0 \mathrm{~b}$ & $0,30 \mathrm{bc}$ & $0,46 \mathrm{bc}$ & $0,34 \mathrm{ab}$ & $0,80 \mathrm{bc}$ \\
7 & 7,4 & $44,0 \mathrm{c}$ & $2,5 \mathrm{~b}$ & $0,03 \mathrm{c}$ & $0,11 \mathrm{c}$ & $0,03 \mathrm{~b}$ & $0,14 \mathrm{c}$ \\
\hline
\end{tabular}

(1) Médias seguidas de mesma letra, nas colunas, não diferem entre si, pelo teste de Tukey, a 5\% de probabilidade.

b) Teste de germinação: foram distribuídas 200 sementes $(8$ repetições de 25 sementes) em rolos de papel toalha mantidos a $30^{\circ} \mathrm{C} \mathrm{e}$ umedecidos em volume de água equivalente a três vezes o seu peso sem hidratação. As avaliações, realizadas aos 21; 28 e 35 dias após a instalação do teste, forneceram a porcentagem de plântulas normais (Brasil, 1992). Diante da ocorrência de poliembrionia na espécie, foi considerado, exclusivamente, um indivíduo para cada semente.

c) Emergência das plântulas: foram semeadas 100 sementes (10 repetições de 10 sementes), a $2 \mathrm{~cm}$ de profundidade, em areia mantida com disponibilidade hídrica próxima à da capacidade de campo, em ambiente sombreado e desprovido de controles de temperatura e de umidade relativa. Foi considerada a taxa (\%) de plântulas que, 42 dias após a instalação do teste, apresentavam a parte área exposta acima da superfície do substrato; da mesma forma adotada no teste de germinação, foi considerado um indivíduo para cada semente.

d) Velocidade de emergência das plântulas: foi obtida, a partir da contagem diária do número de plântulas emersas no teste de emergência das plântulas, através do cálculo de índice segundo Marcos Filho et al. (1987).

e) Comprimentos da raiz, do hipocótilo e da plântula: aos 42 dias após a instalação do teste de emergência das plântulas, foram tomadas, considerando um indivíduo por semente instalada, as distâncias $(\mathrm{cm})$ do ápice da raiz à região de transição com o hipocótilo (comprimento da raiz) e desta à região de inserção das folhas cotiledonares (comprimento do hipocótilo); a soma de ambas as medidas representou o comprimento da plântula. Os dados médios foram obtidos pelo quociente entre o somatório das medidas registradas em cada determinação e o número de sementes utilizadas.

$\mathrm{O}$ delineamento experimental utilizado foi o inteiramente casualizado, e a comparação das médias foi realizada pelo teste de Tukey, ao nível de 5\% de probabilidade; os dados do grau de umidade não foram submetidos à análise estatística.

Adicionalmente, os tratamentos receberam pontuações (Tabela 1) segundo critérios de classificação estatística, fundamentados nas comparações realizadas pelo teste de Tukey, semelhantes aos empregados por Caliari \& Silva (2001); Martins \& Silva (2005) e Martins et al. (2005). Assim, dentro de cada avaliação da qualidade fisiológica, foi atribuída a cada um dos tratamentos a pontuação parcial resultante do somatório das pontuações positivas ou nulas (número de tratamentos estatisticamente superados) com as negativas ou nulas (número de tratamentos estatisticamente superiores); o somatório das pontuações parciais constituiu a pontuação total do tratamento.

\section{RESULTADOS E DISCUSSÃO}

Os graus de umidade determinados, constantes da Tabela 2, apresentaram valores próximos aos desejados e, dessa maneira, indicaram adequação do método de secagem utilizado para obter os tratamentos.

Os dados fisiológicos (Tabela 2), considerando as avaliações de germinação e vigor, indicaram que a desidratação, a partir de $39 \%$ $\mathrm{H}_{2} \mathrm{O}$, tendeu a favorecer o progresso da deterioração das sementes. Contudo, quando confrontados entre si, os testes apresentaram desuniformidade nas comparações de potencial fisiológico entre os tratamentos; essa variação, admitida como resultante de particularidades inerentes à natureza de cada determinação (Grabe, 1976; Marcos Filho et al., 1984), dificultou o estabelecimento de hierarquização dos dados obtidos em todos os testes.

Na busca dessa complementação, os resultados obtidos nos testes fisiológicos foram conjuntamente interpretados, através da

TABELA 3 - Classificação estatística: pontuações parciais (por avaliação) e total (conjunto de avaliações) atribuídas aos tratamentos segundo o desempenho verificado nos testes de germinação (G), da taxa (E) e da velocidade (IVE) de emergência das plântulas, dos comprimentos da raiz $(\mathrm{CR})$, do hipocótilo $(\mathrm{CH})$ e da plântula $(\mathrm{CP})$.

\begin{tabular}{|c|c|c|c|c|c|c|c|}
\hline \multirow{2}{*}{$\begin{array}{c}\text { Tratamentos } \\
\text { (Grau de umidade, \%) }\end{array}$} & \multicolumn{6}{|c|}{ Pontuações parciais por avaliação } & \multirow[t]{2}{*}{ Pontuação total } \\
\hline & $\mathrm{G}$ & $\mathrm{E}$ & IVE & $\mathrm{CR}$ & $\mathrm{CH}$ & $\mathrm{CP}$ & \\
\hline 49 & $(+2)+(0)=+2$ & $(+2)+(0)=+2$ & $(+1)+(0)=+1$ & $(+1)+(0)=+1$ & $(+1)+(0)=+1$ & $(+1)+(0)=+1$ & +8 \\
\hline 39 & $(+4)+(0)=+4$ & $(+2)+(0)=+2$ & $(+4)+(0)=+4$ & $(+3)+(0)=+3$ & $(+1)+(0)=+1$ & $(+2)+(0)=+2$ & +16 \\
\hline 32 & $(+2)+(0)=+2$ & $(0)+(0)=0$ & $(+1)+(0)=+1$ & $(+1)+(0)=+1$ & $(0)+(0)=0$ & $(+1)+(0)=+1$ & +5 \\
\hline 24 & $(0)+(-1)=-1$ & $(0)+(0)=0$ & $(0)+(-1)=-1$ & $(0)+(-1)=-1$ & $(0)+(0)=0$ & $(0)+(0)=0$ & -3 \\
\hline 13 & $(0)+(-3)=-3$ & $(0)+(0)=0$ & $(0)+(-1)=-1$ & $(0)+(0)=0$ & $(0)+(0)=0$ & $(0)+(0)=0$ & -4 \\
\hline 10 & $(0)+(-1)=-1$ & $(0)+(-2)=-2$ & $(0)+(-1)=-1$ & $(0)+(-1)=-1$ & $(0)+(0)=0$ & $(0)+(-1)=-1$ & -6 \\
\hline 7 & $(0)+(-3)=-3$ & $(0)+(-2)=-2$ & $(0)+(-3)=-3$ & $(0)+(-3)=-3$ & $(0)+(-2)=-2$ & $(0)+(-3)=-3$ & -16 \\
\hline
\end{tabular}


atribuição de pontuações (Tabela 3) aos tratamentos, utilizando o critério de classificação estatística aplicado por Caliari \& Silva (2001); Martins \& Silva (2005) e Martins et al. (2005). Nessa classificação, além de haver sido confirmada a tendência previamente observada para a deterioração, o teor de água de $39 \%$, apresentando a maior pontuação total, destacouse dos demais na preservação do potencial fisiológico das sementes. Esse benefício, decorrente da redução moderada do grau de umidade inicial (neste caso, de $49 \%$ para $39 \% \mathrm{H}_{2} \mathrm{O}$ ), foi observado similarmente por Mungomery et al. (1966) e, também, em outras espécies de sementes sensíveis à dessecação (Hor et al., 1984; Boyce, 1989; Gentil et al., 2004).

Assim, a interpretação dos dados sugere que, durante a desidratação, o grau de umidade interfere na deterioração das sementes de tangerina. Graus de umidade superiores a 39\% apresentam menor deterioração; já, nos valores inferiores, o processo da deterioração é favorecido.

\section{CONCLUSÕES}

O potencial fisiológico das sementes de tangerina, considerado o intervalo de 49 a $7 \%$ para o grau de umidade, é favorecido pela desidratação até $39 \% \mathrm{H}_{2} \mathrm{O}$.

\section{AGRADECIMENTO}

Os autores agradecem o Engenheiro Agrônomo Victor Branco de Araújo, da CATI Sementes e Mudas, pela valiosa colaboração para a execução do trabalho.

\section{REFERÊNCIAS}

ARAÚJO, E.F.; CORRÊA, P.C.; PEREIRA, O. A. Influência da temperatura de secagem na germinação de sementes de café. Revista Brasileira de Sementes, Brasília, v.11, n.1-3, p.69-75, 1989.

BARBOZA, R.; HERRERA, J. El vigor en la semilla de café y su relacion con la temperatura de secado, el contenido de humidad y las condiciones de almacienamento. Agronomia Costarricense, Costa Rica, v.14, n.1, p.1-7, 1990.

BOYCE, K.G. Report of the Seed Storage Committee 1986-1989. Seed Science and Technology, Zurich, v.17, sup.1, p.135-142, 1989.

BRASIL. Ministério da Agricultura, do Abastecimento e da Reforma Agrária. Regras para análise de sementes. Brasília: SNAD/DNDV/ CLAV, 1992.365p.

CALIARI, M.F.; SIVA, W.R. Interpretação de dados de testes de vigor na avaliação da qualidade fisiológica de sementes de milho. Revista Brasileira de Sementes, Brasília, v.23, n.1, p.239-251, 2001.

CARVALHO, J.A.; PINHO, E.V.R.V.; OLIVEIRA, J.A.; GUIMARÃES, R.M.; BONOME, L.T. Qualidade de sementes de limão-cravo (Citrus limonia Osbeck) durante o armazenamento. Revista Brasileira de Sementes, Brasília, v.24, n.1, p.286-298, 2002.

CROMARTY,A. S.; ELLIS, R. H.; ROBERTS, E.H. Design of seed storage facilities for genetic conservation. Rome: International Board of Plant Genetic Resources, 1985. 100p.
FARRANT, J.M.; PAMMENTER, N.W.; BERJAK, P. Recalcitrance - a current assessment. Seed Science and Technology, Zurich, v.16, n.1, p.155-166, 1988.

GENTIL, D.F.O.; SILVA, W.R.; FERREIRA, S.A.N. Conservação de sementes de Myrciaria dubia (H.B.K.) McVaugh. Bragantia, Campinas, v.63, n.3, p.421-430, 2004.

GRABE, D.F. Measurement of seed vigor. Journal of Seed Technology, Lansing, v.1, n.2, p.18-31, 1976.

HARRINGTON, J.F. Seed storage and longevity. In: KOZLOWSRI, T.T. (Ed.). Seed biology New York: Academic Press, 1972. v.2. 582p.

HONG, T.D.; ELLIS, R.H. Interspecific variation in seed storage behavior within two genera - coffea and citrus. Seed Science and Technology, Zurich, v.23, n.1, p.165-181, 1995.

HOR, Y.L.; CHIN, H.F.; KARIM, M.Z. The effect of seed moisture and storage temperature on the storability of cocoa (Theobroma cacao) seeds. Seed Science and Technology, Zurich, v.12, n.2, p.415-420, 1984.

KING, M.W.; ROBERTS, E.H. The storage of recalcitrant seeds: achievements and possible approaches. Rome: IBPGR, 1979. 22p.

MARCOS FILHO, J.; PESCARIN, H.M.C.; KOMATSU, Y.H.; DEMÉTRIO, C.G.B.; FANCELLI, A.L. Testes para avaliação do vigor de sementes de soja e sua relação com a emergência das plântulas em campo. Pesquisa Agropecuária Brasileira. Brasília, v.19, n.5, p.605-613, 1984.

MARCOS FILHO, J.; CICERO, S.M.; SILVA, W.R. Avaliação da qualidade das sementes. Piracicaba: FEALQ, 1987.230p.

MARTINS, L.; SILVA, W.R. da; MELETTI, L.M.M. Conservação de sementes de maracujá-amarelo (Passiflora edulis Sims f. flavicarpa Deg). Revista Brasileira de Sementes. Brasília, v.27, n.1, p.183$189,2005$.

MARTINS, L.; SILVA, W.R. da. Interpretação de dados obtidos em testes de vigor, realizados no laboratório e no campo, na comparação da qualidade fisiológica de lotes de sementes de milho. Revista Brasileira de Sementes. Brasília, v.27, n.1, p.19-30, 2005.

MUMFORD, P.M.; PANGGABEAN, G.A. Comparison of the effects of dry storage seeds of Citrus species. Seed Science and Technology, Zurich, v.10, n.2, p.256-266, 1982.

MUNGOMERY, W.V.; AGNEW, G.W.J.; PRODONOFF, E.T. Maintenance of citrus seed viability. Queensland Journal of Agricultural and Animal Sciences, Brisbane, v.23, n.3, p.103-120, 1966.

NAKAMURA, S. The most appropriate moisture content of seeds for their long life span. Proceeding of the International Seed Testing Association, Wageningen, v.3, p.747-759, 1975.

ROBERTS, E.H. Storage environment and the control of viability. In: Viability of the seeds. London: Academic Press, 1972. p.14-58.

TOLEDO, F.F.; MARCOS FILHO, J. Manual das sementes: tecnologia da produção. São Paulo: Agronômica Ceres, 1977. 224p.

VASCONCELOS, L.M.; GROTH, D.; RAZERA, L.F. Efeito de processo de secagem, diferentes graus de umidade e tipos de embalagens na conservação de sementes de café (Coffea arábica L. cv. Catuaí Vermelho). Revista Brasileira de Sementes, Brasília, v.14, n.2, p.181$188,1992$. 